\title{
Trabajo docente en Vaca Muerta: condiciones, sentidos y tensiones desde zonas de sacrificio
}

\author{
Q Ema Paula Penas \\ Universidad Nacional del Comahue (Argentina). \\ paulapenas@hotmail.com \\ María José Laurente \\ Universidad Nacional del Comahue (Argentina). \\ marijolaurente@yahoo.com.ar
}

Fecha de recepción: 16 de abril de 2020.

Fecha de aceptación: 31 de mayo de 2020.

\begin{abstract}
Resumen
En la Norpatagonia argentina desde hace una década se despliega el megaproyecto de fracking Vaca Muerta. Aun cuando las profundas transformaciones territoriales atraviesan el campo educativo, la pregunta sobre qué implicancias tiene educar en estas zonas de sacrificio queda opacada frente a la hegemónica narrativa desarrollista. En base a los estudios sobre trabajo docente y políticas educativas, buscamos describir y comprender las principales condiciones, sentidos y tensiones que se manifiestan en el devenir del quehacer escolar, a partir de cómo sienten y piensan lxs docentes en este enclave petrolero de no convencionales. Desde la lógica cualitativa de la investigación es fundamental escuchar, documentar y analizar las voces de lxs docentes que realizan sus prácticas laborales en estos territorios. Los datos recogidos de entrevistas semiestructuradas se analizan a través del método comparativo constante. En virtud de los silenciamientos estratégicos, la "encerrona constante" y las múltiples tensiones que se producen en el trabajo docente en Vaca Muerta consideramos que - a tono con la dinámica del giro ecoterritorial - la construcción y la difusión de conocimientos sobre estas problemáticas son fundamentales para sensibilizar, problematizar y contribuir a los procesos de resistencia y alternativas al mal desarrollo en América Latina.
\end{abstract}

Palabras clave: trabajo docente; extractivismo; hegemonía; redes de política. 


\title{
Teaching work in Vaca Muerta: conditions, senses and tensions from slaughter areas
}

\begin{abstract}
The Vaca Muerta fracking megaproject has been deployed in Argentina's North Patagonian region for a decade. Even though the region's deep territorial transformations permeate the educational field, the question of what are the implications of educating in these sacrifice zones remains opaque before the hegemonic developmentalist narrative. Based on studies addressing teachers'work and educational policies, and on teachers' perceptions of the dynamics of this unconventional oil enclave, we seek to describe and understand the main conditions, senses and tensions that manifest in the Vaca Muerta's school work. From the qualitative logic of the investigation, it is essential to listen, document and analyze the voices of the teachers who carry out their work practices in these territories. Data collected from semi-structured interviews are analyzed using the constant comparative method. In view of the strategic silencing, the "constant confinement" and the multiple tensions that emerge in the process of teaching in Vaca Muerta we consider that -in keeping with the dynamics of the ecoterritorial turn - the construction and dissemination of knowledge about these problems is fundamental to sensitize, problematize, and contribute to resistance processes to put forward sustainable alternatives to maldevelopment in Latin America.
\end{abstract}

Keywords: teaching work; extractivism; hegemony; political networks.

\section{Introducción}

En una dinámica de veloces transformaciones territoriales y sociales se despliega desde hace unos años Vaca Muerta, un megaproyecto de extracción de hidrocarburos por hidrofractura en Añelo, localidad de la Norpatagonia argentina. A pesar de la relevancia que esta realidad ha adquirido, poco se ha investigado sobre sus aristas en el campo pedagógico, y menos aún en la dimensión del trabajo escolar.

En este artículo indagamos sobre el trabajo que realizan lxs docentes, y las condiciones, sentidos y tensiones que se manifiestan en el devenir de su quehacer en estos particulares territorios perforados por el fracking.

El abordaje desde los estudios sobre trabajo docente y política educativa habilita una mirada dialéctica sobre el espacio laboral en el que confluyen las prácticas hegemónicas, en tensión y conflicto con las luchas, resistencias y propuestas que surgen desde los sectores subalternos. Es fundamental escuchar, documentar y analizar las voces de los trabajadorxs de la educación que realizan sus prácticas laborales en estos territorios de energías extremas a fin de comprender qué piensan y sienten lxs docentes.

\section{Investigar el trabajo docente en zonas de sacrificio}

Este trabajo se inscribe en los resultados de un proyecto de investigación de la Facultad de Ciencias de la Educación de la Universidad Nacional del Comahue, denominado "Políticas educativas, trabajo docente y extractivismo: situaciones de disputa en la Norpatagonia a inicios del siglo XXI (2016-2020)”. En él describimos e interpretamos 
el sentido, la direccionalidad y los fundamentos de aquellas estrategias vinculadas al extractivismo, que ponen de manifiesto lxs sujetxs que disputan las orientaciones de políticas educativas y del trabajo docente.

En torno al extractivismo se producen luchas territoriales, disputas políticas, debates académicos que tensionan los procesos de producción, reproducción social y los paradigmas de vida al diferenciar criterios vinculados a la extracción, apropiación y comercialización de los bienes comunes de la naturaleza. La expansión y legitimación del modelo extractivo se filtran a través de diferentes estrategias y acciones en aristas del espacio educativo.

En la Norpatagonia, estas situaciones son múltiples, variadas y generan profundas tensiones que se manifiestan con mayor claridad en el avance del llamado boom del fracking. El caso paradigmático lo constituye el emprendimiento Vaca Muerta en Añelo, que implica un permanente enfrentamiento de la corporación empresarial (Chevron, YPF, Exxon, Pan American, etc.) con los pueblos originarios, fundamentalmente. La mayoría de los medios de comunicación presenta de manera sistemática el potencial económico y energético de los no convencionales para el desarrollo nacional y la inversión extranjera. Sin embargo, entendemos que son escasas las investigaciones que enfocan cómo el campo educativo forma parte de las transformaciones que se generan en territorios como Añelo, destinado a ser zona de sacrificio. Esta categoría es definida desde la Ecología política latinoamericana para referirse a la delimitación de determinadas áreas geográficas en las que se reciben impactos permanentes y se generan daños ambientales a partir de las acciones de los Estados y las corporaciones, afectando a grupos discriminados y marginados.

El enfoque metodológico se basa en una lógica cualitativa, ya que la misma naturaleza del objeto de investigación exige la progresiva focalización y reformulación del problema durante el proceso investigativo en tanto se pretende identificar la relevancia de los conceptos y las relaciones. El abordaje está motivado por una búsqueda de comprensión de lo social, a través del método de la doble hermenéutica ya que trabaja sobre el significado que lxs investigadorxs le otorgan a las acciones que son significadas por lxs propixs sujetxs. Considera e interroga los dos modos de existencia de lo social, "las estructuras sociales externas, lo social hecho cosas, plasmado en cuestiones objetivas, y las estructuras sociales internalizadas, lo social hecho cuerpo, incorporado al agente" (Gutiérrez, 2005: 16). Atender esta bidimensionalidad permite analizar tanto los hechos observables como las interpretaciones subjetivas enmarcados en un contexto, resguardando la complejidad de los fenómenos sociales.

En el proceso de investigación fue fundamental el trabajo de campo que incluyó la realización de entrevistas semiestructuradas a docentes, preceptorxs y directivxs en sus espacios de trabajo: las dos escuelas primarias y la escuela secundaria de la localidad de Añelo (2019). Dichos datos fueron analizados a través del método comparativo constante. El protocolo inicial de las entrevistas enfoca las particularidades que asume el trabajo docente en la realidad socioterritorial, priorizando las condiciones institucionales-laborales, las articulaciones escuelas/empresas petroleras, el tratamiento pedagógico de las problemáticas relacionadas al fracking y las consideraciones subjetivas sobre el ser docente en Vaca Muerta.

\section{Añelo como territorio con promesa de trabajo}

Esta localidad neuquina fundada en 1915, cuyo nombre significa "médano de la amenaza" o "ciénaga de la muerte" en lengua mapuce, se ubica en una posición estratégica en relación a Neuquén Capital. El inicio, en 2013, de la explotación de hidrocarburos en 
la formación de Vaca Muerta impulsó su reconfiguración territorial transformándose en el centro logístico del sistema productivo regional. La característica de enclave de los modelos extractivos se evidencia en la fragmentación territorial y social plasmada en el paisaje local y en los modos en que ha avanzado la caótica urbanización. Añelo, definida por el BID como capital internacional del Shale (petróleo no convencional), planificada como ciudad sostenible y emergente, es la sede del megaproyecto que concentra las expectativas de un promisorio futuro energético y económico para el país.

\begin{abstract}
Como reflexiona René Zavaletta (2009), asociado a la idea de la naturaleza americana extraordinaria fue cobrando forma el mito del excedente, “uno de los más fundantes y primigenios en América Latina”. Con esta expresión, el autor boliviano hace referencia al mito "eldoradista" que "todo latinoamericano espera en su alma”, ligado al súbito descubrimiento material (de un recurso o bien natural), que genera un excedente, pero un excedente como "magia", "que en la mayor parte de los casos no ha sido utilizado de manera equilibrada”. (Svampa y Viale, 2014: 23)
\end{abstract}

Las investigaciones desde la ecología política latinoamericana cuestionan el carácter colonial y la mirada antropocéntrica sobre los territorios valorados por el capital y analizan la llamada "maldición de la abundancia" (Acosta, 2011) asociando el paradigma extractivista con el empobrecimiento de las poblaciones y el aumento de las injusticias. Otro concepto clave que analiza el derroche y el saqueo del modelo hegemónico es el de maldesarrollo. Svampa y Viale (2014) historizan la categoría destacando que alude a una perspectiva fragmentada, reduccionista y dualista en la cual se viola la integridad de sistemas orgánicos interconectados e interdependientes, y se moviliza un proceso de explotación, desigualdad, injusticia y violencia. La legitimación social de los proyectos y el ocultamiento de las consecuencias que esta dinámica extractivista genera, son posibles con la instalación de una narrativa desarrollista. En ella se articula una dimensión temporal entre memorias, presente y porvenir que opera sobre los horizontes de expectativa social y los sentidos de la vida sosteniendo como premisa que el desarrollo no significa sacrificar nada, sino abrir las puertas al progreso y al crecimiento.

En Neuquén, este vínculo depredador con la naturaleza no es nuevo, ya que su modelo económico es asimilable a un capitalismo de enclave. La tendencia es explotar el recurso natural hasta su agotamiento, utilizando muy poca mano de obra y una distribución de ingresos sumamente inequitativa dependiente de la renta petrolera.

En el marco del boom de los no convencionales en la Argentina se redefinen las claves políticas y económicas de desarrollo de la zona. Fue en este contexto que Añelo, que era un poblado de tan solo 3.000 habitantes, cuadruplicó su población. La instalación del campamento petrolero modifica la vida cotidiana de lxs pobladorxs, incluye nuevxs sujetxs e intereses en la dinámica de ocupación territorial, con una multiplicidad de empresas multinacionales en alianza con los estados provincial y nacional. Las promesas laborales son el eslabón fundamental para ganar la licencia social con la incorporación de trabajadorxs en busca de oportunidades. El pueblo mapuce ofrece una persistente y paradigmática resistencia ya que ve avasallados sus derechos, su identidad y sus territorios; las mujeres mapuces encabezan la batalla acompañadas en algunas luchas por los sectores que defienden el agua y la vida, denunciando la contaminación de los territorios.

\title{
Trabajo docente en Vaca Muerta: las transformaciones en la matrícula escolar como condición laboral (2013-2019)
}

La posibilidad de escuchar las voces de maestrxs y profesorxs, así como sentir y pensar a partir de sus decires permitieron identificar núcleos de sentido que documentamos 
con las referencias empíricas textuales ${ }^{1}$ para interpretarlos en un complejo más amplio de la totalidad histórica. El interrogante central gira en torno a cómo se siente y piensa el trabajo docente en la escuela, partiendo de reconocer que

(...) dada su aparente neutralidad, la escuela parece quedar al margen de la conflictividad socioambiental. A pesar de ser un lugar privilegiado para la construcción de visiones, posiciones y disposiciones sobre el mundo, su estatus en este escenario es atípico, complejo y muchas veces contradictorio. (Escalón Portilla y González Gaudiano, 2017:3)

En cuanto a la particularidad de las condiciones de su quehacer, lxs docentes consideran en primer término el tema de la matrícula escolar; se refieren no solo al aumento de la misma, sino también a los lugares de origen y a los diferentes momentos del año en que se incorporan lxs estudiantxs.

"Un aumento terrible" (D5). Según la Dirección Provincial de Estadística y Censo de Neuquén, ${ }^{2}$ en Añelo (2017) se inscribieron 624 alumnxs en el nivel primario, 227 en el nivel inicial, 314 en el nivel medio, 87 en secundaria de adultos y 5 en primaria especial. En el período 2012-2017 se observa un significativo aumento en la matrícula; la variación es notablemente superior a la de Neuquén, que en el nivel inicial registra un aumento del 14\% mientras en Añelo asciende al 50\%. En primaria los datos marcan también una diferencia importante y el impacto es menos diferencial en la educación secundaria.

"El aumento de la matrícula no está acompañado obviamente de una infraestructura adecuada" (D1). Una cuestión directamente asociada es la dificultad para garantizar la planta funcional que atienda la complejidad institucional. Lxs docentes expresan con claridad: "es bastante complicado" (D1), "estamos peleando" (D4), "los directivos andan batallando para tener los cargos" (D3). Marcan dificultades, presiones y demoras en los nombramientos para ocupar los nuevos puestos de trabajo. Este rasgo remite a la tendencia general de desgaste de la función docente y ajustes de las políticas para la educación pública desde la lógica neoliberal de las reformas educativas globales (Verger, 2012).

Consecuentemente con el aumento de la matrícula, se perciben dificultades en la organización institucional debido a la falta de aulas, cuestión que exige una ardua tarea de readaptación constante del uso del espacio: la ocupación de la biblioteca para otras actividades, el uso de un edificio provisorio para la escuela especial o de un gimnasio alejado de la escuela secundaria. Lxs docentes narran cómo el vínculo con las empresas petroleras pareciera ser la principal vía imaginada para satisfacer las necesidades del funcionamiento y de la arquitectura escolar.

En el caso del secundario asisten estudiantes que viven en las zonas rurales (Sauzal Bonito, Chihuido, Aguada San Roque), y aquí la preocupación se centra en "que no es escuela de doble jornada, y apenas hay un refrigerio a las 9 y a las 15" (D1). Entonces es "una situación riesgosa" el tiempo de estxs estudiantes "que se van y quedan dando vueltas, no tienen acá el control de los padres" (D1). En esta línea se registran testimonios sobre la alimentación y el comedor escolar, en tanto cuestiones básicas de la reproducción social de la vida que se promueve (o no) desde la escuela, profundizando la dimensión del mandato de cuidado y de los compromisos que se espera asuma la docencia.

1 Las citas de entrevistas se referencian manteniendo el anonimato de lxs docentes a través del número de protocolo interno (D\#). 
La multiplicidad de procedencias de lxs estudiantes es otro rasgo que modificó la tradición pueblerina con el arribo de familias atraídas por la promesa laboral.

-Añelo hace 5 años atrás no era en absoluto lo que es ahora y estas situaciones eran mucho menores, esta cuestión de las distintas procedencias, por ejemplo los chicos de República Dominicana, pero también muchos del Norte argentino, de Bolivia, alumnos de Paraguay que han llegado por toda esta situación que se vienen a trabajar 0 al yacimiento o a los servicios. (D1)

La enumeración de las diferencias culturales y lingüísticas se complejiza con el análisis que realiza una de las entrevistadas, cuando - al recuperar las perspectivas críticasenuncia las relaciones de poder, los procesos de estigmatización, racialización, así como las realidades vinculadas a la trata que se viven en Añelo y que llegan a la escuela en la voz de lxs afectadxs.

- Hay muchos niñxs que son dominicanos, eso ha generado el tema del racismo. Tremendo porque también hay un estigma sobre las mamás dominicanas, las mujeres dominicanas son las putas del pueblo. (...) Son tratadas así, yo el año pasado tuve una que me decía: -yo no sé qué hacer, no puedo salir a la calle. Ella trabajaba, pero claro había un nivel de violencia muy fuerte con su hijito porque él reaccionaba frente a "negro de mierda", "andate a tu país" y como que todos estos discursos así medio xenofóbicos y racistas están presentes acá. (D6)

La intensidad del relato se podría asociar con las denuncias e informes judiciales que señalan la existencia de personas víctimas de explotación sexual, entre ellas menores. Las lógicas que imprimen la trata y el racismo en zonas de sacrificio cuando se naturalizan diferentes prácticas extractivas, apuntalan las denominadas pedagogías de la crueldad.

Llamo pedagogías de la crueldad a todos los actos y prácticas que enseñan, habitúan y programan a los sujetos a transmutar lo vivo y su vitalidad en cosas. En ese sentido, esta pedagogía enseña algo que va mucho más allá del matar, enseña a matar de una muerte desritualizada, de una muerte que deja apenas residuos en el lugar del difunto. La trata y la explotación sexual practicadas en estos días son los más perfectos ejemplos y, al mismo tiempo, alegorías de lo que quiero decir con pedagogía de la crueldad.

Es posible que eso explique el hecho de que toda empresa extractivista que se establece en los campos y pequeños pueblos de América Latina para producir commodities destinados al mercado global, al instalarse trae consigo o es, inclusive, precedida por burdeles y el cuerpocosa de las mujeres que allí se ofrecen. (Segato, 2018: 11)

\section{Llegar hasta Añelo: entre las distancias, la canasta petrolera y las luchas sindicales de la docencia}

Otra condición en relación a trabajar en Vaca Muerta es la particular composición del "cuerpo docente", "somos pocos los que somos de Neuquén y menos aún los que se radican en Añelo" (D1). La distancia se convierte en un fuerte condicionamiento, llegan docentes radicadxs tanto en Río Negro como en Neuquén que se trasladan más de $100 \mathrm{~km}$. Dado que menos del 10\% de lxs trabajadorxs de la educación vive en el "pueblo", el transporte diario a cargo del Consejo Provincial de Educación se convirtió en los últimos años en una demanda colectiva y gremial. "Esto del transporte está solucionado hace año y medio recién para media; primaria lo tiene hace un poco más de tiempo, calculo que tres años" (D1). En la historia de esta 
lucha se reconoce que "la única forma de lograrlo fue la permanencia, se logró que no tenemos que poner nuestro auto, nuestro riesgo, nuestro cansancio" (D3b), y en ese punto se reivindica la fuerza de lo colectivo, "a la hora de salir a pelear por algo vamos todos" (D4b).

Este triunfo sindical pareciera potenciar el compromiso individual, reivindicar una acción gremial y afianzar algunos proyectos de trabajo: "viajamos $200 \mathrm{~km}$ todos los días. Desde Cinco Saltos, hace tres años que venimos en transporte y es la gloria. Lo ganamos poniendo el cuerpo" (D3b). La experiencia de lucha, ese "poner el cuerpo" es un hito en la historia de lxs docentes de esa zona.

Los horarios de las traffics agrupan y trasladan a lxs docentes regulando el tiempo de estadía en el pueblo. En estas condiciones, se tiende a tratar de concentrar toda la actividad en la localidad, aún cuando no sean específicamente los puestos más pertinentes al título docente, se recurre a las posibilidades que abren los cargos habilitantes y supletorios.

Los puestos de trabajo en la secundaria son cubiertos por sujetxs con distintas titulaciones: profesorxs, maestrxs de primaria, egresadxs de escuelas técnicas, idónexs o estudiantes con porcentaje aprobado de las carreras docentes. "Esto le da un perfil a la institución" (D1). De todos modos, aún con estas características de reclutamiento, aseguran que "No siempre llegamos a cubrir los cargos, siempre tenemos vacíos, hay cursos que no han tenido clase en determinados trimestres" (D1). El trabajo docente en las escuelas secundarias históricamente ha sido objeto de disputas en torno a las titulaciones, diferentes investigaciones señalan que "la estructura del campo manifiesta un estado de relación de fuerzas entre los agentes e instituciones comprometidas en la lucha por la distribución del capital específico" (Birgin, 1999: 30).

En síntesis las condiciones específicas en las que transcurre el trabajo docente constituyen factores que limitan las dinámicas institucionales y, sobre todo marcan una cierta extranjeridad. "Llegan con el transporte, se van al medio día. No conocen la realidad, la conocen por las noticias, pero no de vivir en el pueblo" (D5).

Quienes no viven en la localidad insisten en plantear como problema principal los elevados precios que se miden en función de la canasta petrolera. Los salarios docentes incluyen un porcentaje por zona desfavorable ${ }^{3}$ que llega al $60 \%$, pero este no basta para que lxs docentes decidan radicarse como nuevxs pobladorxs y, en algunos casos, tampoco es suficientemente atractivo para docentes con experiencia y títulos. El argumento central es el impacto del costo de la vivienda en relación al salario, se hace "imposible vivir acá", "es que el valor de la tierra cotiza como en Puerto Madero" (D6); el sensible desequilibrio que se genera en desmedro de trabajadorxs con menores niveles de ingreso es una razón que explica por qué lxs docentes no viven en Añelo.

Los enclaves de los no convencionales se convierten en territorios de fuertes contrastes, las estimaciones para 2019 planteaban que "el $40 \%$ de los empleos generados, directos e indirectos, será población flotante, es decir, estará parcialmente en el ejido, trabajando, almorzando o en diversas situaciones" (Ministerio del Interior, s./f.). 


\section{Enseñar en la Dubai argentina: hambre, humitos y pobreza}

Lxs entrevistadxs plantean cierta escisión existencial y territorial entre el megaproyecto y la vida escolar, "no lo tenemos identificado como Vaca Muerta, me choca. Es Añelo, lugar de trabajo, no lo vemos con ese nombre de Vaca Muerta” (D3b). Sobre esta relación se tensan y contrastan realidades en simultáneo. La percepción es de antagonismo extremo entre las promesas "eldoradistas" de los no convencionales y las necesidades de la población.

Mientras los medios de comunicación reiteran que Añelo es sinónimo de riqueza y promesa de prosperidad, algunxs docentes perciben una fuerte polaridad: "tanta plata por un lado y tanta necesidad por otro" (D6), "la nueva Dubai de la Argentina y el pibe se me está muriendo de hambre" (D4b). Reconocen cambios contundentes tendientes a desigualdades extremas en las ciudades "frackineadas" a partir de 2013: "La contradicción en la imagen es clara, ves de un lado las casitas con el humito de la leña en el lugar donde se genera la riqueza más codiciada del mundo como es el petróleo y el gas. Afuera la llaman la Dubai de la Patagonia. ¡Minga! Tendrá toda la riqueza pero la gente sigue viviendo en la pobreza, y la escuela pública sigue siendo el escenario más claro para ver eso" (D4a).

En la práctica, el extractivismo ha sido un mecanismo de saqueo y apropiación colonial y neocolonial. Este extractivismo, que ha asumido diversos ropajes a lo largo del tiempo, se ha forjado en la explotación de las materias primas indispensables para el desarrollo industrial y el bienestar del Norte global. Y se lo ha hecho sin importar la sustentabilidad de los proyectos extractivistas, así como tampoco el agotamiento de los recursos. (Acosta, 2011: 2)

Recuerdan lxs docentes que en algún momento hubo comedores escolares, más contención, más presencia del Estado.

El año pasado saltó esta cuestión que era bien palpable de esta gran diferencia de los que consiguieron un trabajito estable en empresas petroleras y los empleados públicos. (...) estábamos en un acto y un nene se descompuso y descubrimos que no había comido, o sea, tenía hambre, se había desmayado por hambre. (D5)

Ese disparador generó organizarse, pero solo se lograron algunas viandas que son vistas como un "parche", "lo que hicimos fue personalizar la situación" (D5), pero ahora varixs chicxs se llevan su comida.

El denominado crecimiento empobrecedor característico de esta lógica de enclave se manifiesta en las dispares situaciones observadas por lxs docentes: "hay muchos vecinos que tienen muy buenos sueldos, pero hay otros que no tienen nada" (D5). En este punto, el economista ecuatoriano Acosta (2011) advierte cómo la sujeción de las situaciones a la lógica del mercado mundial potencia desigualdades y exige anticiparse, en tanto "luego del auge, como consecuencia de la existencia de rigideces para revisar los precios y los salarios, los procesos de ajuste resultan muy complejos y dolorosos" (p. 5). 


\section{Interculturalidad: wenüfoye $e^{4}$ y conflictos socioterritoriales}

En relación a los conflictos socioterritoriales que genera el avance de la frontera extractivista, dentro de las zonas calientes en Vaca Muerta se reconoce la disputa con las comunidades mapuces, concretamente una de las "que está sufriendo las mayores consecuencias de este proceso es Campo Maripe"5 (Alonso y Díaz, 2018: 28). La Confederación Mapuce de Neuquén afirma que el daño cultural que se sufre "a través de la cuestión material ha quebrado la vida comunitaria, porque si perdés todos esos valores comunitarios que te dan identidad basados en el Buen Vivir, en el Kumefelen, desaparecés como cultura" (Scandizzo, 2016: 122).

Algunxs entrevistadxs reivindican la educación intercultural y la centralidad de las comunidades mapuces en el trabajo docente en Añelo. Una de las escuelas primarias se identifica como inclusiva "acá tenemos varias comunidades mapuces (...) las maestras son más combativas están más preocupadas por la cuestión mapuce" (D5). En este aspecto se autodiferencian de la otra escuela por el proyecto de interculturalidad, por los enfoques en los actos escolares, por la presencia de las dos banderas y también por los guardapolvos de algunas maestras que han sido diseñados para mostrar la identificación con la comunidad.

Un hito relevante fue el revuelo que generó el acto escolar del Día de la Bandera en 2017; la prensa nacional titulaba "Polémica en Vaca Muerta: los alumnos de una escuela juraron por 4 banderas". El tema llevó incluso a barajar la amenaza de sumario, frente a la cual el sindicato ATEN (Asociación de Trabajadores de la Educación del Neuquén) se posicionó en defensa de las trabajadoras, al tiempo que se planteaba desde la institución "nosotros revalorizamos el origen de cada uno de los chicos" (D5), y desde esta lógica se presentan diferentes banderas. Lxs entrevistadxs consideran que los medios construyeron en ese contexto "la cuestión mapuce, como enemigo", y vincularon el acto escolar "como postura política, lo relacionaron con Maldonado, ${ }^{6}$ el reclamo de tierras y la necesidad de la presencia de los gendarmes" (D5).

El extractivismo es un modelo que instala una dinámica vertical, expande las fronteras hacia territorios antes considerados improductivos y se expresa a través de megaproyectos. A su paso destruye economías regionales, biodiversidad y profundiza el proceso de acaparamiento de tierras, expulsando o desplazando comunidades rurales, campesinas o indígenas, a la vez que violenta procesos de decisión ciudadana. La dinámica de creciente militarización de los territorios, criminalización de la protesta social y estrechamiento de los límites de la democracia rodea y explica los asesinatos de Santiago Maldonado y Rafael Nahuel ${ }^{7}$ en la Patagonia argentina.

En el contexto de dichas tensiones se posicionan algunxs docentes, "Yo me siento una contradicción (...) venir a enseñar y que te pasen por encima muchas cosas, la problemática con las comunidades mapuces. Pareciera que los chicos y chicas que llegan de las comunidades dejan toda su cosmovisión, su forma de vida al entrar al aula" (D3a).

Según algunxs de lxs entrevistadxs, las dos escuelas primarias parecieran referenciarse como diferentes en torno a la posición política y a la perspectiva sobre la

4 Wenüfoye significa en mapuzungun, la bandera oficializada en 1992 para representar la Nación Mapuce.

5 La Comunidad Mapuce Lof Campo Maripe se ubica geográficamente en el Paraje Vanguardia de la localidad de Añelo, en la Ruta Provincial 17, km 14.

6 Santiago Maldonado fue un joven desaparecido y muerto en agosto de 2017 en el Pu Lof de Resistencia de Cushamen en Chubut, en el marco de la represión de Gendarmería Nacional al corte de ruta llevado a cabo por la comunidad mapuce en reclamo por la recuperación de territorio ancestral.

7 Rafael Nahuel es un joven asesinado en las cercanías del lago Mascardi en noviembre de 2017 durante la represión a la comunidad mapuce Lafken Winkuy Mapu cuando se reclamaba la recuperación de territorio. 
interculturalidad, "con el boom del fracking, una escuela hacia una cosmovisión y otra del otro lado" (D3b). Una docente se autoidenfica planteando que "esta es la escuela de los mapuces, de las feministas o feminazis, de las hippies, de las zurdas, o sea una cantidad de estigmas que han puesto sobre la escuela han sido fuertes en un momento" (D6).

Investigaciones sobre la puesta en práctica de las propuestas interculturales dan cuenta de las diferentes interpelaciones al trabajo docente, y reconocen también cómo algunas "maestras y maestros pueden sostener su tarea frente a tanta complejidad, se las arreglan para entrar en nuevas relaciones de identidad y diferencia" (Díaz, 2001: 137). En síntesis, desde el posicionamiento de algunxs docentes "no es un problema el de la comunidad mapuce; el problema es la cuestión de fondo, del territorio y extractivismo que acá es tremendo" (D6).

\section{¿Fracking?, ¡de eso no se habla!}

Mirar las imágenes de las transformaciones territoriales de la zona de Añelo nos impuso una pregunta para lxs docentes: ¿cómo se presenta el tema del fracking en la vida escolar del principal escenario de los no convencionales de la Argentina?

"De eso no se habla" es tal vez la frase que sintetizó la mayoría de los testimonios; percibimos un cierto recaudo y cuidado para no tensionar la vida institucional en torno a este tema. Esta tendencia incluye el silenciamiento o negación de la cuestión y el uso de tácticas defensivas como "traer la voz antifracking siempre con alguien de afuera porque se complica en lo cotidiano" (D1). En la vida escolar que transcurre sobre Vaca Muerta, el silencio dice mucho, "si lo hablás te vas a terminar peleando. Uno sabe qué es el fracking... pero el sistema te acorrala" (D3a).

La escuela no pareciera ser el escenario privilegiado para alzar la voz frente al fracking. Sin embargo, en la nueva cartografía de las resistencias frente al extractivismo en América Latina se bosqueja una pluralidad de luchas en defensa de lo común que anudan en la centralidad del territorio considerándolo espacio de despliegue de regeneración de la vida con la participación combativa y creativa de las mujeres en estas batallas.

La escuela como singular complejo social, como espacio concreto, es campo de disputa en el cual se superponen mandatos, expectativas, demandas de distintos sectores sociales, es por tanto expresión del mandato estatal (Rockwell, 2000), pero también de las pujas de intereses que se manifiestan en la sociedad civil. Nos preguntamos entonces: ¿qué significan estos silencios estratégicos, esta naturalización del saqueo y el despojo en los propios territorios patagónicos?

\section{La encerrona constante al trabajo docente: donaciones, becas y apoyo desde la alianza estatal-empresarial}

En este punto nos interesa evidenciar cómo penetran capilarmente los intereses extractivistas en el marco de las políticas educativas. Se ponen en juego redes de política, entramados de organizaciones que producen cambios moleculares en las estrategias, formas, relaciones e intereses. Los límites del Estado se ensanchan para fundirse con los de la sociedad civil instalando un modelo de políticas en el que existen cada vez más "elementos de hibridación, entrecruzamiento, límites imprecisos e inestables en los procesos de gobernanza: una mezcla compleja e inestable de jerarquía, heterarquía y mercado" (Ball, 2011: 28). Ya no es el Estado en sentido estricto, el encargado oficial de garantizar los derechos, sino que el 
Estado actúa como broker en tanto opera como articulador entre las instituciones de diversa índole e incita a la privatización de la política. Estos nuevos actores y organizaciones moldean y transforman el sector público de la educación, colaborando en la formación de un nuevo ser en la escuela con nuevos valores, lenguajes y prácticas transmutados del mundo empresarial y conllevan nuevos tipos de relaciones sociales tendientes a la tercerización o la introducción de soluciones con perfil empresarial.

Son variadas las situaciones en las que las empresas y sus fundaciones (Tecpetrol, Panamerican Energy, Total, Schlumberger, YPF) actúan directamente en las instituciones educativas de Añelo: financian la construcción de aulas, proveen diferentes insumos, equipan bibliotecas, donan salas de informática, dictan talleres, ofrecen becas, organizan actividades lúdicas e incluso diseñan Programas de formación docente. En la Norpatagonia, a partir del inicio del fracking, la posición que asumen las petroleras en el sistema educativo se desplaza desde inicios filantrópicos que merodeaban las políticas (Penas, Laurente y Arauz Iussef, 2018), a la participación directa en la gestión. Es decir, se trasladan de la periferia al corazón mismo de las políticas educativas y, de este modo, inciden también en la formación, en la vida laboral y en las subjetividades de la docencia. En estudios actuales de política educativa se resalta cómo el proceso de privatización encubierta es identificado en la relación con otros tipos de empresas, en los cuales la tendencia marca el avance corporativo hacia el corazón de las políticas educativas (Rodríguez y Olivera, 2018).

Las históricas demandas de equipamiento que sufren las escuelas públicas encuentran en Añelo las manos abiertas de la filantropía estratégica de la corporación petrolera. "Una empresa que logramos que ¡nos done las máquinas!, o sea las empresas financian algunas cuestiones con todo lo que ello significa personalmente y también por una cuestión ideológica y política, porque uno apunta a que el Estado sea el garante de la educación pública (...) permiten disponer de insumos o construcción de aulas que son sumamente necesarias para el funcionamiento escolar" (D1).

La dinámica de las lógicas empresariales en torno al fracking precisa de estrategias finamente diseñadas para inmiscuirse en los distintos ámbitos de la ciudadanía. Estas intervenciones están orientadas por múltiples intereses pivoteando sobre la figura de la Responsabilidad Social Empresarial (RSE).

La RSE es una herramienta destinada especialmente a la construcción de una imagen específica de las empresas, que forma parte de la configuración del consenso alrededor de ellas, dentro y fuera de los lugares de trabajo. Esta política se pone en marcha a través de instituciones, con programas específicos, (Figari et al., 2017: 126)

"Es un retema, porque por ejemplo acá las dos aulas que están al final, las construyeron también con esto de la Responsabilidad Social de YPF, después te traen esto de la seguridad vial, alfombrita, una charlita, sacan foto y publican en sus revistas eso" (D6). En lugares clave en los que la alianza estatal-empresarial precisa fortalecer la licencia social, los límites entre lo público y lo privado se diluyen frente a las necesidades de las propias instituciones educativas; desde el decir de lxs docentes, estamos en una "encerrona constante, se renaturaliza la entrada de la empresa a la escuela" (D7).

Frente a las políticas asistencialistas de las multinacionales, lxs trabajadorxs de la educación se muestran presionados entre las necesidades institucionales insatisfechas y las soluciones parciales ofertadas desde las empresas petroleras. La dimensión práctica de la cuestión está atravesada por planteos ético-políticos que caracterizan al trabajo docente. Unx de lxs entrevistadxs esgrime que los fondos son aceptados en pos de satisfacer las necesidades de los chicos, interpretando que las empresas "tienen con nosotros, con la institución una atención" (D5). 
Sin embargo, desde otras perspectivas lxs docentes afirman que "es un lavado de cara al tema del fracking; aceptamos por ausencia del Estado (...) no implica que no sepamos cuál es realmente la función de las donaciones de la ayuda, de los proyectos" (D1). Nos interesa destacar las fuertes y silenciosas implicancias en la conformación de subjetividades y en los silencios estratégicos que presume esta situación para lxs docentes que actúan mediando entre los "beneficios" para lxs niñxs, las familias y las escuelas, a la vez que habilitan el alcance de las acciones de las empresas petroleras.

La pedagogía empresarial del fracking se potencia como educadora para la construcción de hegemonía, consolidando la alianza estatal-empresarial. Se trata de una red de "préstamos" mutuos que estimulan procesos de mercantilización en términos de políticas educativas. Este tipo de acciones de las empresas extractivas en el ámbito escolar no solo son permitidas por el Estado, sino que este las promueve, dando cuenta de la implementación de políticas de subsidiariedad en materia educativa.

Lxs docentes entrevistadxs parecieran aceptar con mayor facilidad aquellas injerencias empresariales vinculadas a la construcción, mantenimiento y dotación de recursos a las escuelas, reconociendo la oportunidad de satisfacer necesidades institucionales frente a la falta de presupuesto estatal. Sin embargo, se muestran reticentes a la penetración de las lógicas empresariales cuando entra en juego la especificidad del trabajo docente pedagógico. Por ejemplo, frente a la implementación de talleres de lectoescritura y de matemática con docentes designados por una universidad privada, en las entrevistas aclaran que "la parte pedagógica se articula con los Jefes de Departamento de la institución" y afirman "sí, nos vamos a oponer fuertemente convencidos de que la función pedagógica es de la institución” (D1).

Las nuevas competencias para lxs trabajadorxs de la educación incluyen la habilidad para realizar contactos con las empresas y aprender a gestionar sus propios presupuestos. "Nosotros pensamos en función de los chicos y no en función de si va a evadir impuestos o no, uno siempre lo hace en función de nuestros alumnos para mejorar los espacios, la calidad. Gracias a Dios siempre hemos tenido respuestas" (D4). El quehacer docente incluye así una constante articulación entre la función asistencial escolar y la RSE.

Las alianzas público-privadas favorecen la desregulación y desprotección del trabajo docente; y el school-based management convierte a los maestros en gestores escolares y a aquellos que trabajan en contextos de vulnerabilidad social se les perfila también como asistentes sociales. (Verger, 2012: 6)

A pesar del fuerte impacto de la pedagogía empresarial, existen fisuras que se expresan en diferentes formas de resistencia. "A algunos como que no les gusta, pero son algunos docentes que ya no están" (D4), así presenta una entrevistada a quienes se negaron a naturalizar la injerencia empresarial quedando anuladas las posibilidades de una confrontación radical. También están aquellxs que aceptan el acceso de las empresas y mantienen una perspectiva de no adhesión política, limitando en parte el juego. "La capacitación estuvo hermosa. Pero en el momento de la foto se levantaron todos y me dejaron solo, ¡ahí hay una resistencia grande para el momento de la foto!" (D4). Se trata de formas que permiten esquivar estratégicamente la utilización del marketing empresarial.

\section{Conclusión}

Si bien los estudios sobre el trabajo docente en contextos de extractivismo aún son escasos, el análisis que realizan Escalón Portilla y González Gaudiano (2017) señala las potencialidades disruptivas de la transgresión selectiva de la institución escolar en 
escenarios de lucha socioambiental. Sin embargo, según nuestros resultados investigativos, lxs docentes entrevistadxs no perciben la escuela como un actor social clave en la resistencia contrahegemónica frente a los megaproyectos de despojos. No obstante, son significativas las disputas pedagógicas y políticas que se libran cotidianamente en la localidad.

El extractivismo define zonas de sacrificio, en las que el trabajo docente y la vida escolar no quedan exentos de las dinámicas de despojo y saqueo territorial. Añelo, presentada por los medios hegemónicos como la Dubai argentina, se encuentra profundamente fragmentada por las promesas "eldoradistas" subsumidas en realidades de pobreza, hambre y necesidades en un ritmo de crecimiento caótico. Desde el sentir-pensar lxs docentes señalan que trabajar en estos particulares territorios afecta las condiciones laborales y redefine la organización institucional tanto por el aumento y la diversidad de la matrícula escolar, como por la complejidad del traslado cotidiano de lxs docentes y estudiantes hasta las escuelas.

Frente a las condiciones edilicias y de equipamiento de la educación pública lxs docentes reconocen algunas situaciones que los ubican en una encerrona constante, alertando sobre las presiones que ejerce el lobby empresarial sobre las escuelas en pos de conseguir la licencia social para el fracking. Allí, desde la alianza estatal-empresarial, las redes de política perfilan estrategias para penetrar el campo pedagógico. Las relaciones asimétricas - características del extractivismo- se replican en el ámbito escolar ubicando a lxs trabajadorxs de la educación entre las necesidades y demandas de la población escolar y la "generosa" filantropía estratégica de la RSE. Esta presión permanente provoca situaciones dilemáticas en las que lxs docentes desarrollan una variedad de respuestas con diferentes grados de adhesión política, generalmente orientadas hacia la convivencia, evitando la confrontación con el megaproyecto. Otra tensión que atraviesa el quehacer educativo está asociada a las concepciones y relaciones con las comunidades mapuce y con los migrantes, cuestión que se encuadra en los límites que el extractivismo marca a las democracias y a las garantías de los derechos humanos.

"De eso no se habla" quizá sea la expresión que mejor sintetiza la elusión de la realidad local que marca el actual vivir sobre la formación de Vaca Muerta. Las resistencias más explícitas a la extracción de los no convencionales devienen de las comunidades mapuces que, a su vez, interpelan el trabajo docente, los proyectos político-educativos y las propuestas de interculturalidad. La reconstrucción hegemónica incluye el aval de un modelo extractivo, el reforzamiento de una única posibilidad de desarrollo junto al individualismo como valor moral radical. Estas tendencias del maldesarrollo son impulsadas indistintamente por las principales fuerzas políticas del país y la provincia, consolidando un cierto conformismo como núcleo del sentido común.

Cuando las clases dominantes intentan demarcar zonas de sacrificio, en las que los despojos múltiples son prácticas habituales que amenazan los derechos humanos y los derechos de la naturaleza, se redefinen las relaciones entre Estado y empresas, trastocando los propios sentidos de la formación y del trabajo docente pedagógico.

La inestabilidad constante sobre el poder económico y simbólico de Vaca Muerta, las movilizaciones en torno a la defensa del agua y el territorio y la información reciente sobre movimientos sísmicos en la zona de Añelo podrían ser algunos de los elementos que jueguen también en la redefinición de la vida escolar y en los sentidos que se le atribuye al trabajo docente en estas zonas calientes. Advertimos que las conclusiones de este trabajo son provisorias y debieran comprenderse en la coyuntura en la que fueron formuladas.

Consideramos que visibilizar y sensibilizar a partir de testimonios de quienes trabajan en esas escuelas es una contribución a las diferentes investigaciones que buscan 
comprender las lógicas extractivas y su afectación a la reproducción social de la vida. En el marco del giro ecoterritorial es urgente producir nuevos conocimientos que promuevan repensar estrategias para articular las gramáticas de lucha de los movimientos socioambientales y de los pueblos originarios, y también del ámbito científico y sindical docente. 


\section{Bibliografía}

"Acosta, A. (2011). Extractivismo y Neoextractivismo: Dos caras de la misma maldición. Revista La línea de fuego. Disponible en: https://lalineadefuego.info/2011/12/23/ extractivismo-y-neoextractivismo-dos-caras-de-la-misma-maldicion-por-albertoacosta/

"Alonso, G. y Díaz, R. (2018). Cuerpo y territorio desde lo alto de una torre: visibilidad, protagonismo y resistencia de mujeres mapuce contra el extractivismo. En Gómez, M. y Sciortino, S. (comps.). Mujeres indígenas y formas de hacer política. Un intercambio de experiencias situadas entre Brasil y Argentina, pp. 27-58. Temperley, Tren en Movimiento.

" Ball, S. (2011). Política social y educativa, empresa social, hibridación y nuevas comunidades discursivas. Propuesta Educativa, Vol. (2), № 36: 25-34, noviembre.

》Birgin, A. (1999). El trabajo de enseñar. Buenos Aires, Troquel.

"Díaz, R. (2001). Trabajo docente y diferencia cultural. Lecturas antropológicas para una identidad desafiada. Buenos Aires, Miño y Dávila.

"Escalón Portilla, E. y González Gaudiano, E. (2017). La escuela como actor social en las luchas contra el extractivismo. Prácticas político-pedagógicas desde la educación comunitaria en Oaxaca, México. Diálogos sobre educación. Temas actuales en investigación educativa, Vol. (8), № 15. Disponible en: http://www.scielo.org.mx/ scielo.php?script=sci_arttext\&pid=S2007-21712017000100004\&lng=es\&tlng=es.

» Figari, C.; Giniger, N.; Palermo, H.; Soul, J.; León Salazar, C.; Hernández, M.; Hirsh, D.; Álvarez Neuman, D.; Cufré, S. y Ciolli, K. (2017). La trama del capital. La hegemonía empresarial en Argentina. Buenos Aires, Biblos.

"Gutiérrez, A. (2005). Las prácticas sociales: una introducción a Pierre Bourdieu. Córdoba, Ferreyra Editor.

"Penas, E.; Laurente, M. y Arauz luseff, M. (2018). Acerca del uso empresarial de las escuelas públicas: entre el extractivismo y la responsabilidad social. Reflexiones desde la Norpatagonia. En VIII Jornadas de Historia de la Patagonia. CURZA. Viedma, Universidad Nacional del Comahue.

》Rockwell, E. (2000). Tres planos para el estudio de las culturas escolares: el desarrollo histórico desde una perspectiva histórica cultural. Interações. Universidad de São Marcos. Vol. (9): 11-25. São Paulo.

»Rodríguez, L. y Olivera, M. (2018). De la periferia al corazón del sistema educativo: cómo el capital construye su proyecto social en Argentina. Los Programas Educativos de las Fundaciones Empresarias. En VII Congreso Nacional y V Internacional de investigación educativa. Facultad de Ciencias de la Educación. Cipolletti, Universidad Nacional del Comahue.

"Scandizzo, H. (2016). Hidrocarburos y diversificación productiva en tiempos de Vaca Muerta. Nueva expansión de frontera del capital Vaca Muerta. Construcción de una estrategia. Buenos Aires, Jinete Insomne.

"Segato, R. (2018). Contra-pedagogías de la crueldad. Buenos Aires, Prometeo Libros.

"Svampa, M. y Viale, E. (2014). Maldesarrollo. La Argentina del extractivismo y el despojo, Buenos Aires, Katz.

»Verger, A. (2012). Globalización, reformas educativas y la nueva gestión del personal 


\section{Documentos citados}

» Ministerio del Interior, Argentina. (s./f.). Informe final Proyecto Ejecutivo para el Mejoramiento Urbano Integral del Centro de Añelo, Neuquén. Disponible en: http://mininterior.gov.ar/planificacion/pdf/planes-doc/NEUQUEN/MejoramientoUrbano-Integral-del-centro-de-A\%C3\%B1elo-parte-I.pdf. Búsqueda 12/12/2019.

\section{Fuentes periodísticas}

»Docentxs sin transporte para llegar a sus escuelas en Añelo reclaman una solución. (16/11/2017). Río Negro. Disponible en: https://www.rionegro.com.ar/docentes-sintransporte-para-llegar-a-sus-escuelas-en-anelo-reclaman-una-solucion-CB3928554/

"El acto por el 20 de junio. Polémica en Vaca Muerta: los alumnos de una escuela juraron por 4 banderas. (21/06/2017). Clarín. Disponible en: https://www.clarin. com/sociedad/polemica-vaca-muerta-alumnos-escuela-juraron-banderas_o_ rkKnrHOmb.html

\section{Ema Paula Penas}

Maestranda en Política y Gestión de la Educación, Universidad Nacional de Luján. Especialista en Pedagogía de la Formación, Universidad Nacional de La Plata; Profesora en Ciencias de la Educación, Universidad Nacional del Comahue. Profesora Regular de Pedagogía en la Universidad Nacional del Comahue. paulapenas@hotmail.com

\section{María José Laurente}

Maestranda en Política y Gestión de la Educación, Universidad Nacional de Luján. Especialista en Elaboración de Diagnósticos para la Formulación de Políticas Educacionales, Universidad Nacional de Luján. Profesora en Ciencias de la Educación, Universidad Nacional del Comahue. Profesora Regular de Política Educacional, Universidad Nacional del Comahue. marijolaurente@yahoo.com.ar 